\title{
Electron-beam-induced annealing of natural zircon: A Raman spectroscopic study
}

\author{
Tamás Váczi ${ }^{1} \cdot$ Lutz Nasdala $^{2}$ \\ $\square \quad$ Tamás Váczi \\ vaczitamas@caesar.elte.hu \\ +3613812297 \\ 1 Department of Mineralogy, Eötvös Loránd University, \\ Pázmány Péter sétány 1/C, 1117 Budapest, Hungary \\ 2 Institut für Mineralogie und Kristallographie, Universität Wien, \\ Althanstr. 14, 1090 Vienna, Austria
}

\begin{abstract}
The annealing of radiation damage in zircon by low-energy electron irradiation was explored systematically. Natural zircon samples spanning a wide range of self-irradiation damage were irradiated with the focused electron beam of an electron probe microanalyser. The effects of beam current and irradiation time were tested systematically, and the changes in zircon were measured using Raman spectroscopy. Our results confirm the damageannealing effect of an accelerated electron beam. We demonstrate that non-thermal annealing occurs through electron-enhanced defect reactions and that the annealing is a function of both the irradiation time and beam current. The complete annealing of radiation damage in zircon by an accelerated electron beam was not possible under the conditions of our experiments. Our results indicate that Raman band broadening in ion-irradiated zircon can possibly be explained through phonon confinement, as the estimated domain sizes of the crystalline volume amid recoil clusters decrease with increasing $\alpha$ dose. The results underlay the importance of doing Raman spectroscopy before electron-beam and ion-beam analysis. To avoid unwanted beam-induced annealing of damage in zircon during EPMA analysis, the electron energy transferred per volume unit of sample should be minimised, for instance by keeping the integrated charge low and/or by defocusing the electron beam.
\end{abstract}

Keywords Zircon $\cdot$ Raman spectroscopy $\cdot$ Band broadening $\cdot$ Radiation damage $\cdot$ Electronbeam annealing 


\section{Introduction}

The electron-probe microanalyser (EPMA) is the most commonly used tool for determining quantitatively the major and minor element chemistry of geomaterials. To generate the X-ray signals, samples are irradiated with a high-current electron beam for an extended time. It is therefore imperative to assess critically the potential changes induced by the beam-material interaction. The electron kinetic energy in an EPMA is typically $15-30 \mathrm{keV}$, which is considered low in materials science. The threshold displacement energies necessary to cause primary knock-on atomic displacements or surface sputtering in thin films (e.g. Jiang and Spence 2009) are in the range of several tens of electronvolts. To overcome the atomic displacement threshold energies, electrons with kinetic energies of at least several hundreds of kiloelectronvolts are needed (e.g. Egerton et al. 2004). However, subthreshold collisions in low-energy electron microscopy samples may be energetic enough to change certain chemical or physical properties of beam-sensitive materials (Egerton et al. 2004; Jercinovic and Williams 2005; Reed 2005 etc.). The specific problem with EPMA X-ray analysis in geosciences, especially of trace constituents such as rare earth elements in zircon, is that it is often essential to work with high electron-dose rates (beam currents of 100-200 nA and above) and long counting times (minutes to tens of minutes) to achieve good X-ray signal count statistics. Cathodoluminescence imaging and spectroscopy may also require prolonged electron exposures.

Materials containing volatile (e.g. $\mathrm{H}_{2} \mathrm{O}$ ) and/or diffusive (e.g. $\mathrm{Na}$ ) components often suffer from beam-induced degradation. This is problematic because of changing analyte compositions in the course of the analysis. The change in chemical composition, however, is not the only way an electron beam may affect the analysed material. Decelerating electrons (see e.g. Egerton et al. 2004) may cause decomposition (radiolysis) as well as sublimation or melting through heating in susceptible materials. Ionisation and redox reactions may also occur through interactions with electrons. The chemical changes and the displacement of atoms by electrons break chemical bonds and may disturb or even destroy the original atomic configuration (short- and long-range order) in the irradiated volume. The collective term commonly used in the literature for material degradation due to external particle-beam bombardment is irradiation damage, or simply beam damage.

Though electron bombardment is generally associated with being a cause for degradation, there are observations that suggest the opposite: the energy delivered by the beam may produce crystallisation, a formation or restoration of long- and short-range order. Crystallisation in amorphous materials has been observed and described mostly in transmission electron microscopy (TEM) studies (e.g. Bae et al. 2007; Meldrum et al. 1997; Utsunomiya et al. 2003). Nasdala et al. (2003), using Raman spectroscopy, demonstrated that EPMA analysis spots are better crystalline than the self-irradiated "matrix" damaged by the $\alpha$ decay of actinides. We can state that even the low-energy electron beam used in EPMA may indeed cause a reconstruction in damaged zircon to a more ordered state (Fig. 1). Note that the backscattered electron (BSE) signal intensity in images of zircon single crystals is correlated with structural damage (Fritzsche and Rothemund 1978; Nasdala et al. 2006, 2007); that is, a lower BSE yield means better crystalline order in a given zircon crystal hosting selfirradiation damage.

Zircon, $\mathrm{ZrSiO}_{4}$, is a common accessory component found in many rocks and geological environments. The actinide impurities naturally incorporated in its structure upon crystal 
growth undergo radioactive decay, producing $\mathrm{Pb}$ isotopes and self-irradiation damage with time. The actinide-Pb isotope systems in zircon are used most often for age determination because of the toughness of the host that often preserves the isotopes and the damage through geologic times. The self-irradiation damage in zircon, caused mostly by $\alpha$ recoil, is fairly straightforward to measure by means of Raman spectroscopy: the broadening (full width at half maximum, FWHM) of the $v_{3}\left(\mathrm{SiO}_{4}\right)$ asymmetric stretching vibration is strongly correlated with the ion irradiation dose (for details see Nasdala et al. 1995, 2001; Palenik et al. 2003). This correlation has been used to assess the structural damage present in zircon (e.g., Nasdala et al. 2001, 2004; Palenik et al. 2003; Zhang et al. 2000b) as well as the equivalent damage level after the partial or full recovery of crystalline order caused by thermal annealing (e.g., Geisler 2002; Geisler et al. 2001; Nasdala and Hanchar 2005; Zhang et al. 2000a).

This article presents a systematic investigation of the effect of electron-beam irradiation on zircon under conditions relevant for electron probe microanalysis. For doing so, the correlation between structural damage and Raman parameters (e.g. Palenik et al. 2003) has been used. The aim of the study is to evaluate the effects of electron-beam irradiation on zircon samples having different levels of initial damage (synthetic fully crystalline to natural heavily damaged), and to explore what the Raman parameter changes infer about the real structure of self-irradiation damaged and annealed zircon.

\section{Materials and methods}

\section{Samples, preparation, and irradiation}

A set of well-characterized, gem-quality zircon samples from Sri Lanka zircon were selected (for a detailed description see Nasdala et al. 2004), to investigate the effect of electron irradiation on different structural states in zircon. A synthetic, pure $\mathrm{ZrSiO}_{4}$ crystal, grown using the Li-Mo flux technique (Hanchar et al. 2001), was used as reference. The samples chosen for this study cover the range from crystalline to severely radiation damaged (see Table 1 and Fig. 2a). Self-irradiation doses were calculated from the present $U$ and Th concentrations and U-Th ages, using the equations of Holland and Gottfried (1955) and Murakami et al. (1991). Following Nasdala et al. (2004), a factor of 0.55 was applied to correct the calculated dose values for the generally and uniformly incomplete (i.e. ca. 55\%) retention of radiation damage in Sri Lankan zircon. Fully amorphous zircon was excluded from the sample set in the present study because low electron energies are apparently insufficient to cause observable changes in completely disordered $\mathrm{ZrSiO}_{4}$ (Meldrum et al. 1997), while at higher electron energies it tends to decompose into oxides rather than undergo zircon crystallisation (e.g. Capitani et al. 2000).

Randomly oriented chips of the gem samples were embedded in epoxy, and ground and polished to produce a high-quality flat surface. The synthetic $\mathrm{ZrSiO}_{4}$ crystal was embedded to reveal a section parallel to its $c$ axis. Polished mounts were given a conductive gold coat in a thermal vacuum evaporator preceding focused ion beam (FIB) marking in a scanning electron microscope (SEM). The thickness of the gold coating was estimated to be ca. $20 \mathrm{~nm}$; this estimate was based on the greenish golden hue of the optically semi-transparent gold layer.

Electron-irradiation spots are most difficult to locate under an optical microscope after the removal of the conductive coating. Therefore, prior to electron irradiation, it was 
necessary to place permanent marks on the surface of the samples to guide the electron beam and to aid the subsequent location of the exact points of irradiation. The $\mathrm{Ga}^{+}$ion beam of a FEI Quanta 3D DualBeam FIB/SEM apparatus (30 kV accelerating voltage, $0.5 \mathrm{nA}$ ion current, fully focused beam, $0.1 \mu$ s dwell time) was used to sputter a $5 \times 5$ grid pattern (dimensions $50 \times 50 \mu \mathrm{m}$ ) in the polished surface of each sample (Fig. 2b). Imaging was done only with the scanned electron beam.

The FIB-marked samples were transferred into a JEOL JXA 8600 Superprobe EPMA for electron irradiation (W cathode, $20 \mathrm{kV}$ accelerating voltage, fully focused beam, Gaussian intensity cross-section verified on secondary electron images). The electron dose-rate (beam current) and the duration of the irradiation (counting time) followed a regular scheme (see Fig. 2c) covering a range of electron irradiations relevant to geochemical analysis. Note that in this paper, we use integrated charge, instead of dose, for the product of beam current and irradiation time. Both the electron dose in a bulk sample and the fluence are quantities problematic to quantify due to the Gaussian cross-section of the beam, the subsurface spread of the interaction volume, and the dependence of beam diameter on the current.

\section{Raman experimental}

A Horiba LabRAM HR UV-Vis-NIR system was used for recording the Raman spectra. The instrument is a dispersive, edge filter-based, confocal Raman microspectrometer equipped with a charge-coupled device (CCD) detector (pixel size $26 \mu \mathrm{m}$ ), which is thermoelectrically cooled to $-70{ }^{\circ} \mathrm{C}$. The $632.8 \mathrm{~nm}$ emission of a He-Ne laser was used for excitation. The confocal pinhole, which functioned also as the spectrograph entrance slit, was set to $50 \mu \mathrm{m}$. An 1800 grooves/mm grating was used in the spectrograph of $800 \mathrm{~mm}$ focal length; the resulting spectral resolution (bandpass, i.e. the FWHM of the instrument profile function) at $\sim 1000 \mathrm{~cm}^{-1}$ Raman shift was $0.6 \mathrm{~cm}^{-1}$. A $100 \times$ microscope objective with a numerical aperture of 0.9 was used to deliver laser light onto the samples and to collect scattered light. The zero-order diffraction position of the spectrograph and the Rayleigh line $\left(0 \mathrm{~cm}^{-1}\right.$ Raman shift) were used for the calibration of the spectrograph dispersion.

Raman point analyses and mappings were done after the removal of the gold coating, through gently wiping coated surfaces using a soft, dry paper tissue. Test measurements through the semi-transparent Au coating failed to give consistently uniform Raman band widths on non-irradiated areas, therefore the removal of the conducting coating was essential.

All samples were individually rotated under the microscope to an orientation that gave the highest intensity of the totally symmetric $v_{3}\left(\mathrm{SiO}_{4}\right) B_{1 g}$ band of zircon. Spots of electron irradiation were located visually under the microscope following the FIB-milled guide marks and post-irradiation imagery. Each point, including non-irradiated blank positions, was measured three times by repeatedly positioning the confocal focal spot. Exposure times were $2-10 \times 15$ s for natural samples and $2 \times 10$ s for synthetic $\mathrm{ZrSiO}_{4}$. The two-dimensional Raman map of $40 \times 46$ pixels was recorded using $2 \times 20$ s exposure time per point, $0.4 \mu \mathrm{m}$ step size. The three-dimensional (3D) Raman map was created by mapping an area covered by $38 \times 20$ pixels ( $0.2 \mu \mathrm{m}$ step size) on the sample surface; the same area was scanned in eight depth levels, each $0.5 \mu \mathrm{m}$ apart, focusing progressively deeper into the sample. The exposure time was $2 \times 9$ s per sampling point.

For precise band position correction, Ne glow-discharge light from a calibration lamp was introduced through the Raman microscope using the transmission illumination path. As a result, a Ne atomic emission line $\left(671.704 \mathrm{~nm}, 14887.5 \mathrm{~cm}^{-1}\right.$, at $914.9 \mathrm{~cm}^{-1}$ apparent Raman 
shift) appeared on each recorded single spectrum. Zircon Raman band positions were corrected by the difference between the measured position and the reference value of this $\mathrm{Ne}$ line. The position of the Ne line is sufficiently close to the studied $v_{3}\left(\mathrm{SiO}_{4}\right)$ zircon Raman band; therefore, any potential inaccuracy in the calibration or the linearity of the spectrograph can be ignored. The accuracy of reported zircon band positions in point measurements is determined mostly by the scatter between individual measurements (Table 1). The main source of the scatter is presumed to be sample variability and the inaccuracy of repeatedly positioning Raman point measurements precisely at the irradiation spots.

\section{Raman-band fitting and deconvolution}

The PeakFit software was used to fit analytical curves to measured point spectra. Instead of assigning a baseline in a separate step, baseline and spectral bands were fitted together to the experimental spectrum to reach the best overall fit, with the aim of minimising the bias due to baseline subtraction. Since the FWHMs of fitted profiles are sensitive to the exact form of the baseline, the same type of baseline (hyperbolic) was used for all spectra.

The bandpass of the spectrometer was confirmed to approximate a Gaussian profile by control measurements: $100 \%$ pure Gaussian shapes were reported by no-constraint pseudoVoigt (weighted Gaussian-Lorentzian sum) profile fits to the Rayleigh line $\left(0 \mathrm{~cm}^{-1}\right)$ as well as to two He-Ne laser plasma lines appearing at $c a .961$ and $1008 \mathrm{~cm}^{-1}$ apparent Raman shifts (632.8 $\mathrm{nm}$ excitation). The Gaussian width parameter $\sigma=\mathrm{FWHM}_{\text {Gaussian }} /[2 \sqrt{ }(2 \ln 2)]$ of the spectrometer bandpass was determined on the two plasma lines $\left(\sigma=0.25 \mathrm{~cm}^{-1}\right.$ was used in zircon Raman band fitting).

Zircon Raman bands [ $v_{1}$ and $\left.v_{3}\left(\mathrm{SiO}_{4}\right)\right]$ were fitted using the Voigt function in the area form that returns deconvolved Gaussian $\sigma$ and Lorentzian $\gamma$ width parameters ("Voigt Area G/L” function in PeakFit). The experimentally determined $\sigma$ value of the spectral bandpass (see above) was used for both Raman bands in the Voigt profiles. These Gaussian widths were "locked" (i.e., not allowed to refine) during peak fitting. Thus, the software adjusted the baseline, the band positions and amplitudes but only the Lorentzian half-widths $(\gamma)$. This procedure directly yielded bandpass-deconvolved Raman band half-widths. An essentially analogous method for bandpass deconvolution using non-commercial software was described by Sundius (1973). To create maps of Raman band widths, Gaussian-Lorentzian weighted sum functions were fitted to the Raman bands in the spectrometer's LabSpec 5 software, and the deconvolution of the instrumental broadening was done subsequently using the numerical approximation formula of Váczi (2014).

\section{Monte Carlo simulations}

Particle-matter interactions were predicted mathematically using Monte Carlo (MC) methods. Electron-energy transfer and the interaction volume were simulated using CASINO 3.2 (Demers et al. 2011). Two million trajectories were calculated with default interaction parameters in a $\mathrm{ZrSiO}_{4}$ substrate having a user-defined density of $4.7 \mathrm{~g} \mathrm{~cm}^{-1}$. The number and distribution of atomic displacements as well as the penetration depths of ions in a $\mathrm{ZrSiO}_{4}$ target were simulated using the detailed damage-cascade calculation option in the SRIM-2013 software (Ziegler et al. 2010). Displacement energies of $75 \mathrm{eV}$ for $\mathrm{Zr}, 75 \mathrm{eV}$ for Si and $60 \mathrm{eV}$ for O (Moreira et al. 2009), SRIM default values for binding energies, and a target density of $4.7 \mathrm{~g} \mathrm{~cm}^{-1}$ were used. 


\section{Results}

\section{Raman spectroscopic observations}

The mean of the $v_{3}\left(\mathrm{SiO}_{4}\right)$ Raman FWHM data from non-irradiated ("blank") cells were compared to the mean of one hundred independent FWHM measurements in non-irradiated areas. The difference between the mean values was not more than $0.2 \mathrm{~cm}^{-1}$ for any given selfirradiated sample, verifying that blank measurements served as reliable reference FWHM values (Table 1). In the case of sample syn the FWHM difference between any measurements was below $0.1 \mathrm{~cm}^{-1}$.

The synthetic reference sample (syn) showed no evident Raman FWHM change after electron irradiation. We can safely state that fully ordered zircon suffers no beam-induced change to its crystallinity under low-energy electron beams. For this reason, synthetic zircon will not be discussed any further in this paper.

In contrast, all self-irradiated zircon samples showed a decrease in the measured $v_{3}\left(\mathrm{SiO}_{4}\right)$ Raman FWHM after low-energy electron irradiation, hence reconfirming earlier findings (Nasdala et al. 2003). Figure 3 shows the Raman map of a ca. $16 \times 18 \mu \mathrm{m}$ area of the sample with the lowest initial damage level in this study (M144). Spots of decreased FWHM reveal the electron irradiation sites (shown as "hills" in Fig. 3), indicating that electron irradiation increased the crystalline order. Ion-milled crosses (FIB marks) show increased FWHM values, which however cannot be explained directly by knock-on damage created by the Ga beam. The stopping range of $30 \mathrm{keV} \mathrm{Ga}^{+}$ions is approximately $16 \mathrm{~nm}$, modelled using the SRIM-2013 software (Ziegler et al. 2010). Such a thin skin of damage in not expected to yield an observable increase in the measured FWHM because the volume sampled by a Raman microscope in a transparent medium is almost one magnitude larger (see Nasdala 2009). The exact cause of the FWHM increase at the sites of Ga ion beam sputtering is yet unknown.

The spatial distribution of the changes in Raman properties was mapped in three dimensions as well. Figure 4a shows a depth section through the centre of the $200 \mathrm{nA}, 500 \mathrm{~s}$ irradiation spot in sample M144. For comparison, the absorbed electron energy was simulated using the CASINO Monte Carlo code (Demers et al. 2011). A beam of $0.5 \mu \mathrm{m}$ diameter and a Gaussian intensity profile was modelled. A $10 \mathrm{~nm}$ thick slice was calculated through the beam centre, the energy cut-off for trajectory simulation was set to $50 \mathrm{eV}$ (Fig. 4b). We found the largest decrease in the FWHM to be nearest to the surface, confirming that focusing the Raman excitation at the sample surfaces allowed measuring the volume of maximum annealing. The oversampled Raman map in Figure 4a may be considered as the convolution of the confocal Raman volume with the electron interaction volume in Figure 4b. Though volume deconvolution was not attempted, it appears that very low electron energies, perhaps as low as $100 \mathrm{eV}$, are able to produce a decrease in the $v_{3}\left(\mathrm{SiO}_{4}\right)$ Raman FWHM.

The fitted, deconvolved ("true") Raman FWHM values of point measurements recorded at electron irradiation spots in self-irradiated samples are shown in Figure 5. The same data are represented in three ways to aid discussion. On Figure 5a, the results are shown as column charts (category $x$ and $y$ axes) to compare numerical FWHM data. However, these charts distort time vs. FWHM and beam current vs. FWHM relationships. Therefore, Figure $5 \mathrm{~b}$ and c show surface plots with proportional $x$ and $y$ axes (irradiation time and beam current, 
respectively). One set of surface plots compares the FWHM recovery in the four samples (Fig. 5b) while the other set shows the absolute band width changes on a common FWHM scale (Fig. 5c).

The results shown in Figure 5 reveal that the larger the beam current (dose rate) or the longer the irradiation time is, the larger is the decrease in the measured zircon Raman FWHM values. A very important observation is, however, that irradiations with similar integrated electron charges (see Fig. 2c) cause similar decreases in FWHM. This is best seen in the G4 sample data, since the FWHM decrease is the largest and the scatter in measured values represent the smallest relative error. This observation is also direct experimental evidence showing that the FWHM decrease in bulk zircon is not caused by electron beam heating at 20 $\mathrm{kV}$. We do not see any enhancement of the FWHM recovery at large beam currents compared to equivalent integrated charges at lower currents. Essentially the same behaviour was observed by Meldrum et al. (1997) during moderate-energy (200 keV) electron irradiation of a $\mathrm{LaPO}_{4}$ thin film. They found that identical electron doses were needed to reach the same crystallisation state and a larger beam current (dose rate) did not change the necessary crystallisation dose.

In addition to direct experimental evidence on the absence of thermal annealing, we also estimated the maximum temperature rise during electron irradiation. The estimation was made using Eq. 3.196 of Reimer (1998):

$$
\Delta T=3 P(1-\eta) /(2 \pi \kappa R),
$$

where $P$ is the beam power, $\eta$ is the backscattering coefficient, $\kappa$ is thermal conductivity, and $R$ is the electron range (see also Liu et al. 1994). The following values were used in Eq. 1 for the calculation of the temperature rise: $20 \mathrm{kV}$ accelerating voltage, $200 \mathrm{nA}$ beam current, $\eta=$ 0.41 (from a CASINO simulation of $\mathrm{ZrSiO}_{4}$ with a conductive gold coat of $20 \mathrm{~nm}$ thickness), thermal conductivity values of $\kappa=3.9 \mathrm{~W} \mathrm{~m}^{-1} \mathrm{~K}^{-1} \perp c$ and $4.8 \mathrm{~W} \mathrm{~m}^{-1} \mathrm{~K}^{-1} \| c$ from Clauser and Huenges (1995), and $R=2.76 \mu \mathrm{m}$ electron range in the continuous slowing-down approximation (calculated for $20 \mathrm{keV}$ using the NIST ESTAR online tool, http://physics.nist.gov/PhysRefData/Star/Text/ESTAR.html). The equation predicts a temperature rise in the range of 105-85 K for crystalline zircon. A surface layer of high thermal conductivity is known to decrease the heat transfer into the bulk of the sample significantly (Jercinovic and Williams 2005). The presence of the gold coating on our samples predicts that the maximum temperature rise most probably remained below $100 \mathrm{~K}$ at the centre of the irradiated spot, even under the largest beam current in this study. Bulk sample heating or, in other words, thermal annealing therefore can be excluded.

The FWHM decrease does not scale linearly with the integrated charge, as evident from a comparison of charts showing the integrated charges (Fig. 2c) and FWHMs (Fig. 5). While not obviously manifest on column charts (Fig. 5a), surface plots (Fig. 5b-c) clearly show that the slopes of the FWHM decrease flatten as the beam current and the irradiation time get progressively larger. The same pattern, with remarkable similarity, can be followed in all samples containing $\alpha$-irradiation damage (Fig. 5b). The magnitude of the change at any given integrated charge (Fig. 5c) is positively correlated to the initial FWHM, and hence to the initial damage inferred from the estimated effective $\alpha$-decay damage levels (Table 1).

To evaluate the evolution of the Raman parameters during electron irradiations, we plotted corrected band positions against FWHM values (Fig. 6a). Sample M144 follows approximately the reverse of the damage accumulation trend. Sample OR1 appears to have shifted only horizontally, i.e., their band positions changed very little along with the decrease 
of the FWHM. Sample M146 appears to be intermediate between M144 and OR1. The Raman parameters of sample G4 behave remarkably different in comparison: the band positions shift to higher values at small integrated charges but then reverse towards lower Raman shifts above ca. $200 \mathrm{nA}$ for $100 \mathrm{~s}$ or equivalent integrated charges, while the FWHMs continuously decrease (Fig. 6a). For comparison, Figure 6b shows the evolution of the zircon Raman parameters during thermal annealing (from Geisler et al. 2001).

\section{Accuracy of data}

The experimental methods applied in this study are associated with certain difficulties that allow a semi-quantitative evaluation of results. Raw materials included one pure, synthetic $\mathrm{ZrSiO}_{4}$ crystal and well-characterised, homogeneous zircon gemstones. This permitted a quantitative estimation of $\alpha$ doses and Raman parameters in non-irradiated samples (Table 1; see also Nasdala et al. 2004). The electron beam of the microprobe was started and stopped manually (by means of mouse clicks in the operating software), beam live times are therefore estimated to be accurate within $0.5 \mathrm{~s}$. Apparently the largest error was caused by the uncertainty of positioning Raman measurements exactly at the electron irradiation sites. Placing a Raman point measurement off the best position causes a slight underestimation of the change of the FWHM. The guide marks and the post-irradiation images of the contamination rings on sample surfaces were essential in locating the right spots.

Nevertheless, the necessary removal of the conductive coating, and the contamination rings with it, created difficulties in the precise positioning of Raman measurements. Note that sites of long irradiations do occasionally show slightly increased specular reflectivity. The locations of Raman measurement spots are estimated to be accurate within $0.5 \mu \mathrm{m}$ of the FWHM minimum position.

Another source of error is the inhomogeneity measureable in the polished samples. This may come from "intrinsic" inhomogeneity (zoning, inclusions), but generally all samples were selected to be free of such phenomena. Another possible source is sample preparation: strain from breaking gemstones into small chips or remnant dislocations from deep scratches during rough grinding. Note, however, that strain should be seen in the band position rather than in FWHMs (Nasdala et al. 2008). Our opinion, based on the detailed analysis of out-oftrend spots, is that outlier data points (Fig. 5a) are most probably artefacts from sample preparation.

\section{Discussion}

\section{Raman band broadening}

The broadening, position shift and asymmetry of Raman bands are usually very apparent in ion-irradiated materials, including self-irradiated and artificially ion-irradiated zircon. The measurable FWHM as well as the position and the intensity of the $v_{3}\left(\mathrm{SiO}_{4}\right)\left(B_{1 g}\right)$ mode, found at $1008 \mathrm{~cm}^{-1}$ in crystalline zircon, is known to correlate with the ion dose and, hence, with the amount of radiation-induced damage in the crystalline lattice. The correlation was established on naturally radiation-damaged, unannealed zircon samples (Nasdala et al. 1995, 2001), but the same correlation is often extended as a direct measure of damage to zircon material 
partially annealed (e.g. Geisler et al. 2001; Nasdala et al. 2004; Zhang et al. 2000a; this study), ion-irradiated with light ions (Nasdala et al. 2005, 2011) and so on.

Palenik et al. (2003) and Nasdala et al. (2004) related an extended range of corrected self-irradiation $\alpha$ dose in zircon to Raman band broadening. The model function fitted to the Raman data by Palenik et al. (2003) is slightly inaccurate as their model curve starts at $0 \mathrm{~cm}^{-1}$ Raman FWHM at zero damage. Since zircon with no damage has an intrinsic band width of $1.8-1.9 \mathrm{~cm}^{-1}$ at room temperature (Nasdala et al. 2002; this study), a slightly revised damage accumulation curve, fitted to the annealing-corrected data of Palenik et al. (2003), of the form

$$
v_{3}\left(\mathrm{SiO}_{4}\right) \mathrm{FWHM}=A_{1}-A_{2} e^{-B_{\mathrm{FWHM}} D}
$$

is suggested here (Fig. 7a), where $A_{1}$ and $A_{2}$ are scaling factors $\left(A_{1}=34.96 \mathrm{~cm}^{-1}\right.$ is the asymptote at large $\alpha$ doses $D$, and $A_{2}$ is chosen such that $A_{1}-A_{2}=1.8 \mathrm{~cm}^{-1}$, equivalent to the FWHM offset at zero dose); $B_{\text {FWHM }}=5.32 \times 10^{-19} \mathrm{~g} / \alpha$ is the increment per $\alpha$ event. The modified trend (Eq. 2) will be used in this study to assess the FWHM vs. dose relationship.

\section{Periodic domain sizes}

The $\alpha$ decay of actinides and daughter isotopes causes most of the stable self-irradiation damage in natural (e.g. Ewing et al. 2003) and Pu-doped synthetic zircon (Weber 1991). One proxy for the evaluation of accumulating damage is the estimation of the amorphous fraction $\left(f_{\mathrm{a}}\right)$, that is, the mass fraction of material amorphised by decay events. X-ray diffraction (XRD) and ${ }^{29}$ Si magic-angle spinning nuclear magnetic resonance (MAS NMR) are two experimental methods that may be used to estimate the amorphous fraction (see e.g. Farnan and Salje 2001; Ríos et al. 2000). Molecular dynamics (MD) simulations can suggest recoil sizes and the number of permanent atomic displacements (e.g. Devanathan et al. 2006), while a geometrical model of recoil connectivity may be used to link single events and bulk properties including the amorphous fraction (Ketcham et al. 2013).

The model of Ketcham et al. (2013) provided undamaged domain sizes as well, through which it became, to date, the only source of information on the possible dimensions of crystalline lattice remnants within self-irradiated zircon. The mean intercept length, $l_{\text {int }}$ (the length of probe segments divided by the number of intersections with the "amorphous" domains), was used by Ketcham et al. (2013) to estimate the size of crystalline remnants. We extrapolated the intercept length in the 8-length chain model $\left({ }^{238} \mathrm{U}\right.$ decay) to large doses using the formula

$$
l_{\text {int }}=4.6295 \times 10^{18} \mathrm{D}^{-1},
$$

which is plotted as a function of "recoil" dose in Figure 7a. The mean intercept length in the model of Ketcham et al. (2013) is only one of the many possible ways to estimate the dimensions of undamaged volumes (R.A. Ketcham, pers. comm.).

However, one must exercise some caution when using the Ketcham et al. (2013) model. When building the connectivity model, the input values for the dimensions of the amorphous "capsules" were scaled to reproduce the amorphous fraction evolution estimate published by Palenik et al. (2003). These authors converted their Raman FWHM vs. dose plot into an amorphous fraction evolution curve. Unfortunately, Raman spectroscopy is not directly suitable for the estimation of the amorphous fraction, contrary to the approach used by Palenik et al. (2003). In insulator crystals, the dominant Raman interaction is phonon scattering; that is, the major contribution comes from the crystalline fraction (specifically, the 
volume segments possessing defect-free translational order). The crystalline fraction $\left(f_{\mathrm{c}}\right)$ is not fully complementary to the amorphous fraction (i.e., $f_{\mathrm{a}}+f_{\mathrm{c}}<1$ ), because there exist a transitional, defect-rich fringe around recoil clusters (see e.g. Devanathan et al. 2006). The damage created in single recoil events was therefore heavily overestimated by Palenik et al. (2003) but was adopted without change by Ketcham et al. (2013). The damage per $\alpha$ event calculated following Palenik et al. (2003) $\left(1.96 \times 10^{22}\right.$ atoms $/ \mathrm{g} \times 5.48 \times 10^{-19} \mathrm{~g} / \alpha \approx 10800$ displaced atoms per $\alpha$ event) is approximately double of the value inferred from the results of Ríos et al. (2000) or Farnan and Salje (2001) (ca. 5300 atoms/ $\alpha$ event).

It is challenging, or even impossible without the reconstruction of the model, to predict the effects of the underestimation of the event sizes on percolation thresholds and intercept lengths, and it is not the purpose of this contribution. Nevertheless, the size estimates for undamaged domain sizes laid out by Ketcham et al. (2013) are most probably in the correct order of magnitude, which allows us to draw preliminary, qualitative conclusions on the effects of electron-beam annealing on zircon. To indicate the unknown uncertainty in domain sizes (Eq. 3), we used thick lines for the curves showing domain size evolution in Figure 7, and the plots should be regarded only as rough guides for discussion. Furthermore, the intercept lengths reported in the connectivity model are mean values. The size distributions of translational domains are currently unknown, and may not be best represented by the mean. This aspect is currently under investigation (for an approximate analogy in diamond nanocrystals, see Osswald et al. 2009).

Figure 7 compares the revised Raman broadening model curve (í. 2) and the mean intercept length in the geometric percolation model of Ketcham et al. (2013) (Eq. 3). Figure 7 suggests very small "crystalline" domains in radiation-damaged zircon: the largest modelled dose, $0.19 \times 10^{18} \alpha$ recoil/g, corresponds to $22.8 \mathrm{~nm}$ as the mean domain size (Ketcham et al. 2013). In the extrapolated range, the trend suggests periodic domains of approximately $5 \mathrm{~nm}$ at a dose of $1 \times 10^{18} \alpha$ recoils/g, and ca. $1 \mathrm{~nm}$ at $4.5-5 \times 10^{18} \alpha$ recoil $/ \mathrm{g}$. The small dimensions of coherently vibrating domains may easily be the cause for the observed band broadening through phonon confinement in damaged zircon (cf. Geisler and Pidgeon 2002).

\section{Annealing}

An initial drop in the $v_{3}\left(\mathrm{SiO}_{4}\right)$ Raman band width and its gradually decreasing rate of change upon further irradiation is a general trend across samples (Fig. 5b-c). This suggests that the annealing kinetics at different levels of radiation damage bear certain similarities: a portion of the damage is annealed apparently easily, while the reordering of the rest of the damage appears progressively more inhibited. The FWHMs change at flatter rates at elevated electron doses, which may reflect that the more strongly disordered environments do not anneal as easily, that is, the further increase of ordered domains become progressively more inhibited, regardless of the initial damage level.

The changes in FWHM (cf. Table 1) as well as initial and final estimated crystalline domain sizes, following the model of Ketcham et al. (2013), are listed in Table 2 and marked on Figure 7b. Remarkably, the changes in FWHM are not proportional to the changes in the estimated periodic domain size, since these two properties follow different trends. Note that the mean domain size may indicate a shift of the maximum as well as a change in the size distribution (see above).

It is not possible to conclude without doubt, but seems likely, from the current set of experiments and results that damage cannot be annealed completely under a low-energy 
electron beam. To test this issue unambiguously, very large irradiation doses, far in excess of those applied in this study, would be needed. We assume that in these samples the totally disordered volume fragments, that is, the amorphous cores of recoil clusters, remain largely unaffected by electron-beam annealing at the energy and doses used in this study. This is indicated by the Raman spectral parameters: residual band broadening is observed in all samples even after the irradiation with the largest dose (Fig. 5b). The apparent stability of amorphised volumes under electron irradiation is supported by the observations of Meldrum et al. (1997), who did not see the epitaxial growth of the crystalline part in zircon under a TEM beam at 80-160 keV electron energies. Note that other materials do exhibit epitaxial recrystallization under an electron beam (e.g. $\mathrm{LaPO}_{4}$ : Meldrum et al. 1997; $\mathrm{SrTiO}_{3}$ : Zhang et al. 2005; silicate apatite: Bae et al. 2007), therefore this behaviour must be specific to the given structure or composition. A further evidence for the absence of the recrystallization of amorphous domains is that it is readily possible to image recoil clusters in an electron microscope (Bursill and McLaren 1966; Capitani et al. 2000; Lian et al. 2003; Meldrum et al. 1997; Murakami et al. 1991; Palenik et al. 2003; Wang and Ewing 1992; Weber et al. 1994; Zhao et al. 2010). Nevertheless, in the light of the results of this study, it seems highly likely that some rearrangement of defect atoms does occur in zircon during the interaction with the electron beam in a TEM as well.

The annealing of defects involves the migration of atoms and/or vacancies. Point defects undergo diffusion, through which they may cluster, migrate to crystallite surfaces, or vacancies and interstitials may recombine and annihilate. Amorphous domains may undergo epitaxial recrystallization onto preserved crystalline fragments (Geisler 2002; Nasdala et al. 2002; Zhang et al. 2000a etc.), or, especially in extended amorphous volumes, may undergo spinoidal decomposition (see Váczi et al. 2009 and references therein). The mechanism behind the electron-beam-stimulated annealing of defects is a mobility of interstitial ions and vacancies, driven by the non-thermal energy transfer from accelerated electrons to the material. Zhang et al. (2005) suggested that incident electrons primarily cause ionisations and produce localised electronic excitations, which affect chemical bonds and may lower activation energies for defect reactions. Specific vibrational modes may be bound to localised electronic states (Balkanski 1986) that may be excited by the electron irradiation. According to Weeks et al. (1975), the recombination of excited electron-hole pairs release energy that is converted mostly into vibrational energy (phonons). These phonons, localised around the defects, may promote defect reactions. Nevertheless, we can assume that a non-thermal activation of the mobility of defects and the promotion of defect reactions (hopping of defects, annihilation etc.) takes place under electron irradiation.

A comparison of thermal and electron-beam induced annealing reveals that the recovery pathways are not identical (Fig. 6). Thermal annealing of "partially metamict" zircon was interpreted to progress in two stages: the first stage was assigned to a recovery of order in volumes retaining a degree of crystallinity, while the second stage comprises the epitaxial recrystallization of completely amorphised zircon (e.g., Geisler et al. 2001; Weber et al. 1994; Zhang et al. 2000a). Interestingly, the two stages of annealing are apparent in the evolution of the upshift of the $v_{3}\left(\mathrm{SiO}_{4}\right)$ Raman band, whereas the FWHM displays a smooth decrease (Fig. 6b; see also Geisler et al. 2001; Zhang et al. 2000a). In our study, in agreement with the observations of Meldrum et al. (1997), it may be assumed that the epitaxial growth of the crystalline volume did not occur. In the lack of epitaxial growth during electron irradiation, the mechanism that can increase phonon coherence lengths is most probably the annealing of defects in the crystalline volume fragments and at the crystalline-to-amorphous interfaces. 
Based on the different behaviour of the Raman parameters during electron-beam driven and thermal annealing (Fig. 6), however, we can state that the two ordering processes are not identical.

\section{Conclusions}

In zircon damaged by ion irradiation, the crystalline domain sizes appear to decrease with increasing $\alpha$ dose, and rapidly reach very small dimensions. Consequently, it is plausible to state that the confinement of phonons due to the decrease of coherent domains is the cause of observed Raman broadening in ion-irradiated zircon.

The observed decrease of the $v_{3}\left(\mathrm{SiO}_{4}\right)$ Raman FWHMs in zircon is attributed to the electron irradiation-induced migration and annihilation of crystallographic defects that act as phonon scattering centres. The assumed ordering apparently creates a gradual increase in the periodic domain sizes and, therefore, a decrease in the observable Raman FWHMs. In zircon with little damage, small changes in Raman FWHMs may signal a large recovery in domain sizes. At increasingly larger damage levels, in spite of the marked decrease of Raman FWHMs, the recovery of crystalline domain sizes may actually be rather small. The trend of levelling-off in the rate of FWHM change observed in our experiments suggest that some degree of crystallinity (i.e., an at least partially ordered template) is needed for low-energy electron irradiation to anneal defects, as well as that recoil clusters do not anneal in the time frame of several hundred seconds.

Since lattice strain and phonon confinement (both contributing e.g. to the shift of Raman band positions) may not be convincingly decoupled in radiation-damaged materials, it is difficult to draw all-embracing conclusions on the evolution of the crystalline phase (in terms of size, defect density and distribution) based only on Raman spectroscopic parameters. Further study is in progress to gain more insight into the real structure during the accumulation and the annealing of radiation damage in zircon.

Finally, we may formulate a few recommendations on working with radiation-damaged materials using electron-beam analysis. Most importantly, the best practice is to perform Raman spectroscopy before electron microscopy (especially X-ray analysis and CL imaging/spectroscopy), whenever possible. A non-prolonged scanning of the beam is not expected to cause observable changes even under a rather strong beam current; the integrated charge (time $\times$ current) is the deciding factor. Point analyses, however, may anneal the sample, and, therefore, cause a mobility of incompatible elements, within 10-20 seconds. If the sample allows (i.e., in the lack of fine zoning or other short-scale inhomogeneity), the analyses should be done with as much beam defocus as is feasible. In contrast to all the caution necessary when analysing natural samples, the phenomenon described in this paper may actually be beneficial in creating shallow waveguides (Babsail et al. 1991) with customtailored geometry in $\mathrm{ZrSiO}_{4}$ using double irradiation. The crystalline-to-amorphous interfaces in ion-irradiated materials may be sharpened using large-dose electron irradiation in order to improve the waveguide's performance.

Acknowledgements Gem zircon samples were kindly made available by Wolfgang Hofmeister (Mainz) and Allen K. Kennedy (Perth), and the synthetic zircon crystal was provided by John M. Hanchar (St. John’s). Andreas Möller (Lawrence, KS) is thanked for the 
permission to use the Rogaland sample mounts in the present study. Sample preparation was done by Andreas Wagner (Vienna). We gratefully acknowledge the experimental assistance by Nora Groschopf (Mainz), Theodoros Ntaflos (Vienna), Ferenc Kristály and Norbert Zajzon (Miskolc), Reinhard Kaindl (Innsbruck), Gábor Varga, Zsolt Bendő and Péter Horváth (Budapest). Richard A. Ketcham is thanked for comments. We are indebted E. Schweizerbart Science Publishers for the permission to reproduce Fig. 6b. T.V. expresses his thanks for access to the research infrastructure in the Core Facility for Research and Instruments, Faculty of Science, Eötvös University (Budapest). This project has been supported by the National Research, Development and Innovation Office of Hungary grant no. OTKA PD116183 to T.V., and by the Austrian Science Fund (FWF) grant no. P24448-N19 to L.N.

\section{References}

Babsail L, Hamelin N, Townsend PD (1991) Helium-ion implanted waveguides in zircon. Nucl Instrum Meth B 59:1219-1222. doi: 10.1016/0168-583X(91)95797-H

Bae IT, Zhang YW, Weber WJ, Higuchi M, Giannuzzi LA (2007) Electron-beam induced recrystallization in amorphous apatite. Appl Phys Lett 90:021912. doi: 10.1063/1.2430779

Balkanski M (1986) Dynamics of localized phonon modes. In: Butcher PN, March NH, Tosi MP (eds) Crystalline Semiconducting Materials and Devices. Springer US, Boston, MA, pp 195-216

Bursill LA, McLaren AC (1966) Transmission electron microscope study of natural radiation damage in zircon $\left(\mathrm{ZrSiO}_{4}\right)$. Phys Stat Sol B 13:331-343. doi: 10.1002/pssb.19660130205

Capitani GC, Leroux H, Doukhan JC, Ríos S, Zhang M, Salje EKH (2000) A TEM investigation of natural metamict zircons: structure and recovery of amorphous domains. Phys Chem Mineral 27:545-556. doi: 10.1007/s002690000100

Clauser C, Huenges E (1995) Thermal conductivity of rocks and minerals. In: Ahrens TJ (ed) Rock Physics \& Phase Relations: A Handbook of Physical Constants, vol 3. American Geophysical Union, Washington, D. C., pp 105-126

Demers H, Poirier-Demers N, Couture AR, Joly D, Guilmain M, de Jonge N, Drouin D (2011) Threedimensional electron microscopy simulation with the CASINO Monte Carlo software. Scanning 33:135-146. doi: 10.1002/sca.20262

Devanathan R, Corrales LR, Weber WJ, Chartier A, Meis C (2006) Molecular dynamics simulation of energetic uranium recoil damage in zircon. Mol Simul 32:1069-1077. doi: $10.1080 / 08927020600959929$

Egerton RF, Li P, Malac M (2004) Radiation damage in the TEM and SEM. Micron 35:399-409. doi: 10.1016/j.micron.2004.02.003

Ewing RC, Meldrum A, Wang L, Weber WJ, Corrales LR (2003) Radiation effects in zircon. In: Hanchar JM, Hoskin PWO (eds) Zircon /Rev Mineral Geochem 53/. Mineralogical Society of America, Washington, D.C., pp 387-425

Farnan I, Salje EKH (2001) The degree and nature of radiation damage in zircon observed by ${ }^{29} \mathrm{Si}$ nuclear magnetic resonance. J Appl Phys 89:2084-2090. doi: 10.1063/1.1343523

Fritzsche CR, Rothemund W (1978) Investigation of radiation damage by electron beam absorption measurements. Appl Phys 16:339-343. doi: 10.1007/BF00885857

Geisler T, Pidgeon RT, Van Bronswijk W, Pleysier R (2001) Kinetics of thermal recovery and recrystallization of partially metamict zircon: a Raman spectroscopic study. Eur J Mineral 13:1163-1176. doi: 10.1127/0935-1221/2001/0013-1163

Geisler T (2002) Isothermal annealing of partially metamict zircon: evidence for a three-stage recovery process. Phys Chem Mineral 29:420-429. doi: 10.1007/s00269-002-0249-3 
Geisler T, Pidgeon RT (2002) Raman scattering from metamict zircon: comments on "Metamictisation of natural zircon: accumulation versus thermal annealing of radioactivity-induced damage" by Nasdala et al. 2001 (Contribution to Mineralogy and Petrology 141: 125-144). Contrib Mineral Petrol 143:750-757. doi: 10.1007/s00410-002-0378-1

Hanchar JM, Finch RJ, Hoskin PWO, Watson EB, Cherniak DJ, Mariano AN (2001) Rare earth elements in synthetic zircon: Part 1 . Synthesis, and rare earth element and phosphorus doping. Am Mineral 86:667-680

Holland HD, Gottfried D (1955) The effect of nuclear radiation on the structure of zircon. Acta Crystallogr 8:291-300. doi: 10.1107/S0365110X55000947

Jercinovic MJ, Williams ML (2005) Analytical perils (and progress) in electron microprobe trace element analysis applied to geochronology: Background acquisition, interferences, and beam irradiation effects. Am Mineral 90:526-546. doi: 10.2138/am.2005.1422

Jiang N, Spence JCH (2009) Radiation damage in zircon by high-energy electron beams. J Appl Phys 105:123517. doi: 10.1063/1.3151704

Ketcham RA, Guenthner WR, Reiners PW (2013) Geometric analysis of radiation damage connectivity in zircon, and its implications for helium diffusion. Am Mineral 98:350-360. doi: 10.2138/am.2013.4249

Lian J, Ríos S, Boatner LA, Wang LM, Ewing RC (2003) Microstructural evolution and nanocrystal formation in $\mathrm{Pb}^{+}$-implanted $\mathrm{ZrSiO}_{4}$ single crystals. J Appl Phys 94:5695-5703. doi: 10.1063/1.1618917

Liu M, Xu LY, Lin XZ (1994) Heating effect of electron beam bombardment. Scanning 16:1-5. doi: 10.1002/sca.4950160102

Meldrum A, Boatner LA, Ewing RC (1997) Electron-irradiation-induced nucleation and growth in amorphous $\mathrm{LaPO}_{4}, \mathrm{ScPO}_{4}$, and zircon. J Mater Res 12:1816-1827. doi: 10.1557/JMR.1997.0250

Moreira PAFP, Devanathan R, Yu JG, Weber WJ (2009) Molecular-dynamics simulation of threshold displacement energies in zircon. Nucl Instrum Meth B 267:3431-3436. doi:

10.1016/j.nimb.2009.07.023

Murakami T, Chakoumakos BC, Ewing RC, Lumpkin GR, Weber WJ (1991) Alpha-decay event damage in zircon. Am Mineral 76:1510-1532

Nasdala L, Irmer G, Wolf D (1995) The degree of metamictization in zircon: a Raman spectroscopic study. Eur J Mineral 7:471-478

Nasdala L, Wenzel M, Vavra G, Irmer G, Wenzel T, Kober B (2001) Metamictisation of natural zircon: accumulation versus thermal annealing of radioactivity-induced damage. Contrib Mineral Petrol 141:125-144. doi: 10.1007/s004100000235

Nasdala L, Lengauer CL, Hanchar JM, Kronz A, Wirth R, Blanc P, Kennedy AK, Seydoux-Guillaume A-M (2002) Annealing radiation damage and the recovery of cathodoluminescence. Chem Geol 191:121-140. doi: 10.1016/S0009-2541(02)00152-3

Nasdala L, Zhang M, Kempe U, Panczer G, Gaft M, Andrut M, Plötze M (2003) Spectroscopic methods applied to zircon. In: Hanchar JM, Hoskin PWO (eds) Zircon /Rev Mineral Geochem 53/. Mineralogical Society of America, Washington, D.C., pp 427-467

Nasdala L, Reiners PW, Garver JI, Kennedy AK, Stern RA, Balan E, Wirth R (2004) Incomplete retention of radiation damage in zircon from Sri Lanka. Am Mineral 89:219-231. doi: 10.2138/am-2004-0126

Nasdala L, Hanchar JM (2005) Comment on: Application of Raman spectroscopy to distinguish metamorphic and igneous zircon (Xian et al., Anal. Lett. 2004, v. 37, p. 119). Anal Lett 38:727734. doi: 10.1081/AL-200050353

Nasdala L, Hanchar JM, Kronz A, Whitehouse MJ (2005) Long-term stability of alpha particle damage in natural zircon. Chem Geol 220:83-103. doi: 10.1016/j.chemgeo.2005.03.012 
Nasdala L, Kronz A, Hanchar JM, Tichomirowa M, Davis DW, Hofmeister W (2006) Effects of natural radiation damage on back-scattered electron images of single crystals of minerals. Am Mineral 91:1739-1746. doi: 10.2138/am.2006.2241

Nasdala L, Kronz A, Grambole D, Trullenque G (2007) Effects of irradiation damage on the backscattering of electrons: Silicon-implanted silicon. Am Mineral 92:1768-1771. doi: 10.2138/am.2007.2648

Nasdala L, Miletich R, Ruschel K, Váczi T (2008) Raman study of radiation-damaged zircon under hydrostatic compression. Phys Chem Mineral 35:597-602. doi: 10.1007/s00269-008-0251-5

Nasdala L (2009) $\mathrm{Pb}^{+}$irradiation of synthetic zircon $\left(\mathrm{ZrSiO}_{4}\right)$ : Infrared spectroscopic investigationDiscussion. Am Mineral 94:853-855. doi: 10.2138/am.2009.541

Nasdala L, Grambole D, Götze J, Kempe U, Váczi T (2011) Helium irradiation study on zircon. Contrib Mineral Petrol 161:777-789. doi: 10.1007/s00410-010-0562-7

Osswald S, Mochalin VN, Havel M, Yushin G, Gogotsi Y (2009) Phonon confinement effects in the Raman spectrum of nanodiamond. Phys Rev B 80:075419. doi: 10.1103/PhysRevB.80.075419

Palenik CS, Nasdala L, Ewing RC (2003) Radiation damage in zircon. Am Mineral 88:770-781. doi: 10.2138/am-2003-5-606

Reed SJB (2005) Electron Microprobe Analysis and Scanning Electron Microscopy in Geology. Cambridge University Press, Cambridge

Reimer L (1998) Scanning Electron Microscopy. Springer, Berlin, Heidelberg

Ríos S, Salje EKH, Zhang M, Ewing RC (2000) Amorphization in zircon: evidence for direct impact damage. J Phys: Condens Mat 12:2401-2412. doi: 10.1088/0953-8984/12/11/306

Sundius T (1973) Computer fitting of Voigt profiles to Raman lines. J Raman Spectrosc 1:471-488. doi: 10.1002/jrs.1250010506

Utsunomiya S, Yudintsev S, Wang LM, Ewing RC (2003) Ion-beam and electron-beam irradiation of synthetic britholite. J Nucl Mater 322:180-188. doi: 10.1016/S0022-3115(03)00327-1

Váczi T, Nasdala L, Wirth R, Mehofer M, Libowitzky E, Häger T (2009) On the breakdown of zircon upon "dry" thermal annealing. Mineral Petrol 97:129-138. doi: 10.1007/s00710-009-0087-9

Váczi T (2014) A new, simple approximation for the deconvolution of instrumental broadening in spectroscopic band profiles. Appl Spectrosc 68:1274-1278. doi: 10.1366/13-07275

Wang LM, Ewing RC (1992) Detailed in situ study of ion beam-induced amorphization of zircon. Nucl Instrum Meth B 65:324-329. doi: 10.1016/0168-583X(92)95060-5

Weber WJ (1991) Self-radiation damage and recovery in Pu-doped zircon. Radiat Eff Defect Solid 115:341-349. doi: 10.1080/10420159108220580

Weber WJ, Ewing RC, Wang LM (1994) The radiation-induced crystalline-to-amorphous transition in zircon. J Mater Res 9:688-698. doi: 10.1557/JMR.1994.0688

Weeks JD, Tully JC, Kimerling LC (1975) Theory of recombination-enhanced defect reactions in semiconductors. Phys Rev B 12:3286-3292. doi: 10.1103/PhysRevB.12.3286

Zhang M, Salje EKH, Capitani GC, Leroux H, Clark AM, Schluter J, Ewing RC (2000a) Annealing of $\alpha$-decay damage in zircon: a Raman spectroscopic study. J Phys: Condens Mat 12:3131-3148. doi: 10.1088/0953-8984/12/13/321

Zhang M, Salje EKH, Farnan I, Graeme-Barber A, Daniel P, Ewing RC, Clark AM, Leroux H (2000b) Metamictization of zircon: Raman spectroscopic study. J Phys: Condens Mat 12:1915-1925. doi: 10.1088/0953-8984/12/8/333

Zhang Y, Lian J, Wang CM, Jiang W, Ewing RC, Weber WJ (2005) Ion-induced damage accumulation and electron-beam-enhanced recrystallization in $\mathrm{SrTiO}_{3}$. Phys Rev B 72:094112. doi: 10.1103/PhysRevB.72.094112

Zhao GP, Treacy MMJ, Buseck PR (2010) Fluctuation electron microscopy of medium-range order in ion-irradiated zircon. Philos Mag 90:4661-4677. doi: 10.1080/14786431003630876

Ziegler JF, Ziegler MD, Biersack JP (2010) SRIM - The stopping and range of ions in matter (2010). Nucl Instrum Meth B 268:1818-1823. doi: 10.1016/j.nimb.2010.02.091 
Table 1 Estimated self-irradiation doses and fitted $v_{3}\left(\mathrm{SiO}_{4}\right)$ Raman parameters from blank measurements (see Figure 1). Each reported Raman parameter is given as the mean of fifteen independently measured values \pm the maximum probable error

\begin{tabular}{lccc}
\hline Sample & $\begin{array}{c}\text { Effective } \boldsymbol{\alpha} \text { dose } \\
{[\mathbf{1 0} \boldsymbol{\mathbf { 1 8 }} \mathbf{g}]}\end{array}$ & $\begin{array}{c}\text { FWHM } \\
{\left[\mathbf{c m}^{-\mathbf{1}}\right]}\end{array}$ & $\begin{array}{c}\text { Raman shift } \\
{\left[\mathbf{c m}^{-\mathbf{1}} \mathbf{]}\right.}\end{array}$ \\
\hline syn & 0 & $1.9 \pm 0.0$ & $1008.2 \pm 0.4$ \\
M144 & $0.47-0.50$ & $7.5 \pm 0.5$ & $1003.9 \pm 0.5$ \\
M146 & $1.06-1.12$ & $14.0 \pm 0.6$ & $1000.2 \pm 0.5$ \\
OR1 & $1.44-1.61$ & $21.5 \pm 1.1$ & $996.5 \pm 0.6$ \\
G4 & $2.46-2.70$ & $28.3 \pm 1.1$ & $997.4 \pm 0.7$ \\
\hline
\end{tabular}

Following Nasdala et al. (2004), $\alpha$ doses calculated from U, Th concentrations and U-Pb ages were multiplied by 55\%, to account for partial damage annealing experienced by the Sri Lanka zircon

Table 2 Observed changes of (corrected) FWHMs of the $v_{3}\left(\mathrm{SiO}_{4}\right)$ Raman band (see also Table 1) and corresponding, estimated mean periodic domain sizes before and after electron irradiations at the maximum integrated charge in this study (200 nA for $500 \mathrm{~s}$ )

\begin{tabular}{lccc}
\hline Sample & FWHM change $\left[\mathbf{c m}^{\mathbf{- 1}}\right]$ & $\boldsymbol{l}_{\text {int }}$ before $[\mathbf{n m}]$ & $\boldsymbol{l}_{\text {int }}$ after $[\mathbf{n m}]$ \\
\hline M144 & -1.4 & 13.1 & 17.9 \\
M146 & -3.3 & 5.3 & 7.8 \\
OR1 & -4.0 & 2.7 & 3.9 \\
G4 & -7.1 & 1.5 & 2.8 \\
\hline
\end{tabular}



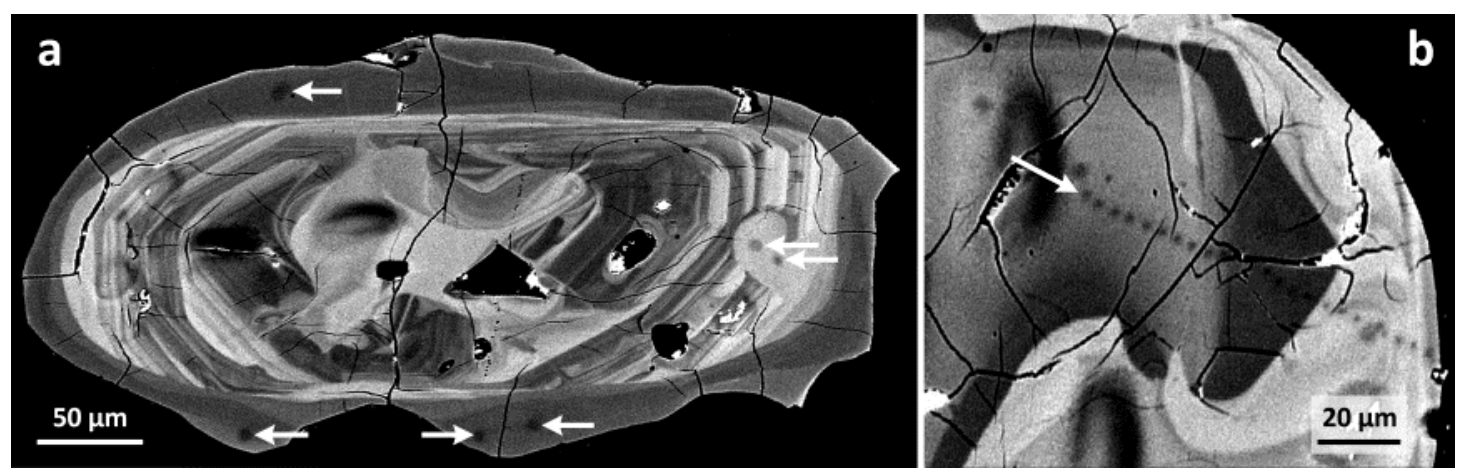

Fig. 1 Two zircon grains from the ultrahigh-temperature granulites of Rogaland, Norway (for sample descriptions, see Möller et al., 2003). The back-scattered electron (BSE) images, taken after EPMA measurements, show darkened spots at the sites of electron irradiation $(20 \mathrm{keV}, 100 \mathrm{nA}, 2 \mu \mathrm{m}$ spot diameter). The images show scattered analysis spots (1800 s per spot, left image) and a trace of a line scan (180 s per spot, right image). Samples courtesy A. Möller
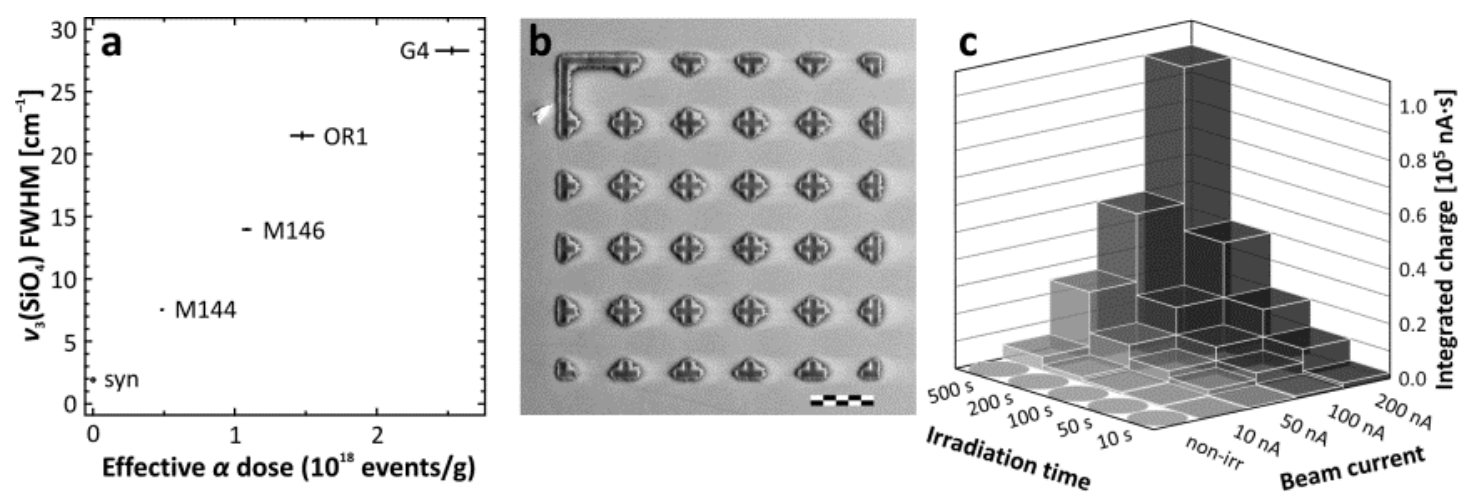

Fig. 2 Sample selection and preparation. a Plot of FWHM vs dose of the samples used in this study. b Guide marks on the surface of sample M144 after FIB-milling (secondary electron image). The length of the scale bar is $5 \mu \mathrm{m}$. c Chart of the electron doses, expressed as the product of time and beam current, irradiated into the 25 cells shown in $\mathbf{b}$

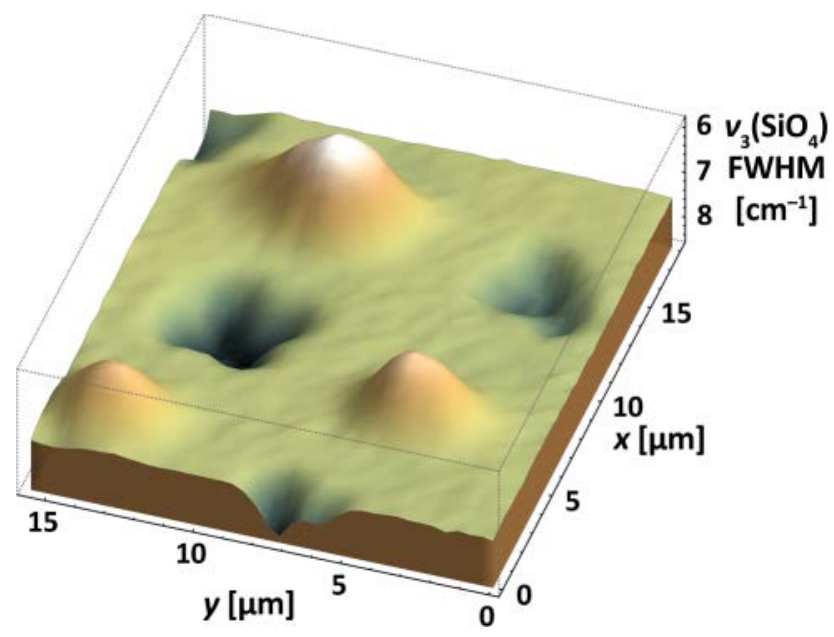

Fig. 3 Raman map of an area of sample M144, constructed using bandpass-deconvolved $v_{3}\left(\mathrm{SiO}_{4}\right)$ FWHM values. "Peaks" show decreased FWHMs at the sites of electron irradiation, while FIB marks are seen as “depressions”. (Front left: 100 nA, 200 s; front right: 100 nA, 500 s; back: 200 nA, 500 s) 


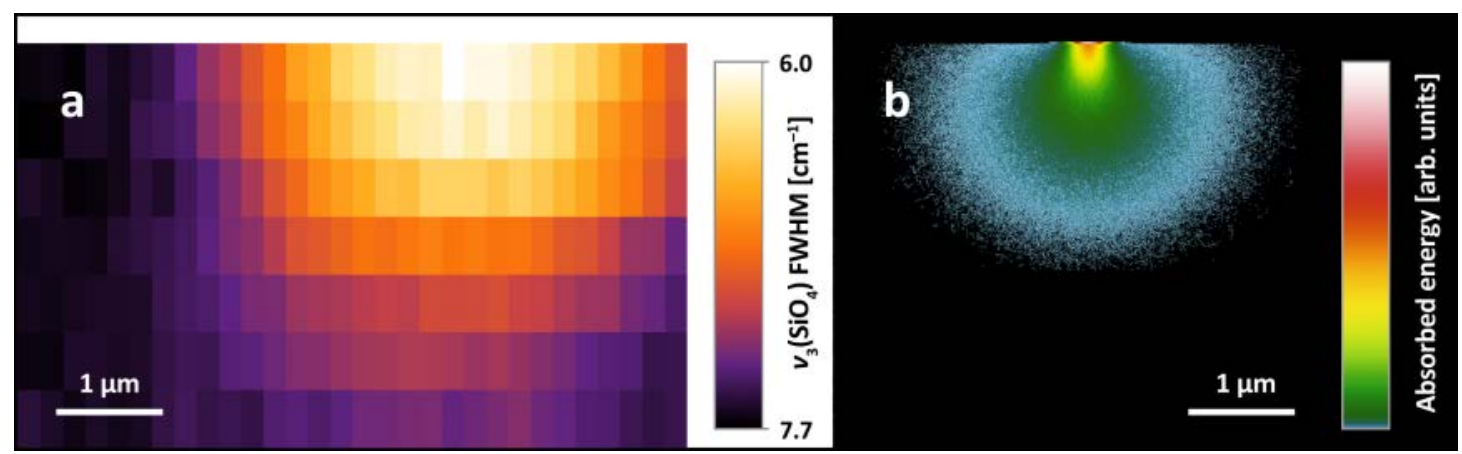

Fig. 4 Spatial distribution of the change in the $v_{3}\left(\mathrm{SiO}_{4}\right)$ Raman FWHM, and of the electron interaction volume. a Depth section constructed from a 3D Raman FWHM map $(0.2 \mu \mathrm{m}$ horizontal, $0.5 \mu \mathrm{m}$ vertical step size) through the highest-dose electron irradiation (200 nA, $500 \mathrm{~s}$ ) in sample M144. b Monte Carlo simulation showing the absorbed energy in a $10 \mathrm{~nm}$ thick slice through the centre of the irradiating electron beam. The energy cut-off (change from light blue to black) is at $50 \mathrm{eV}$
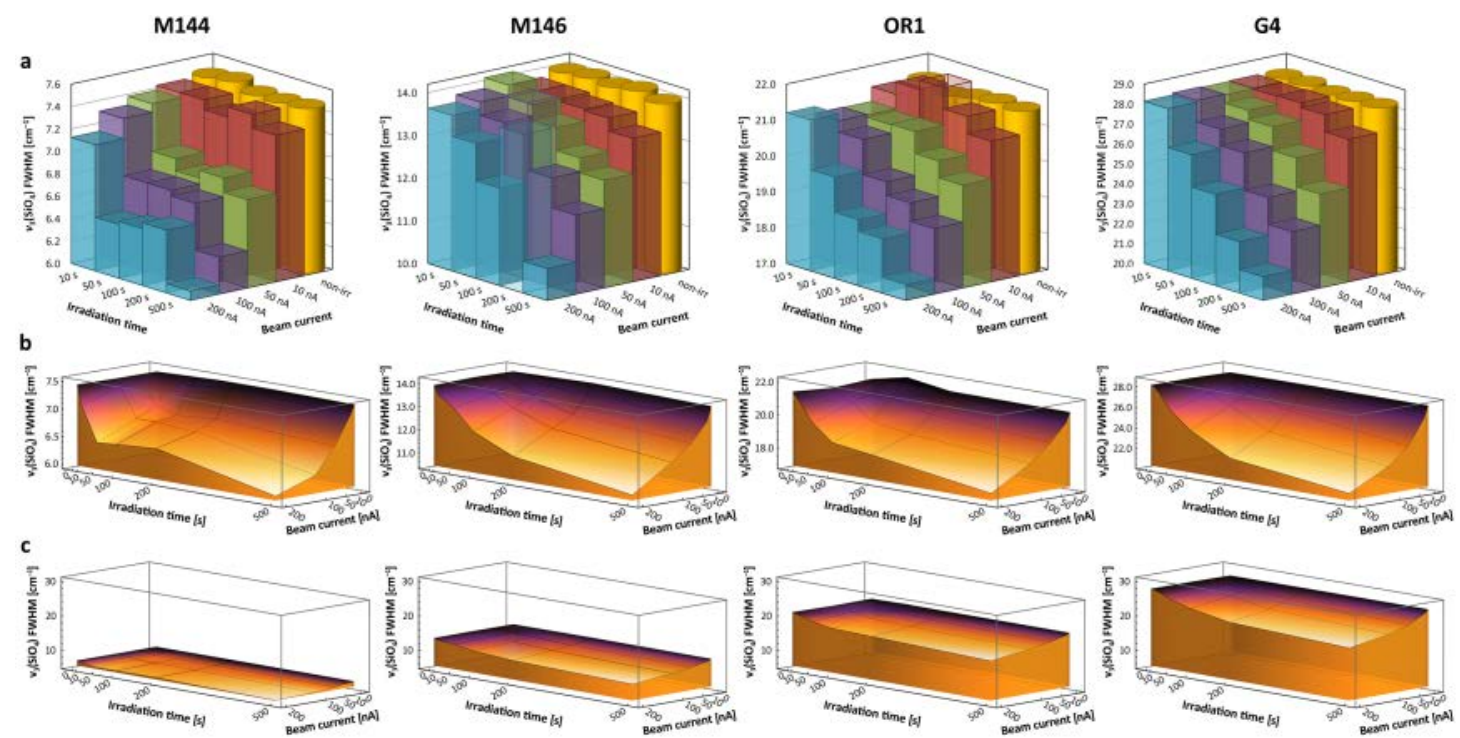

Fig. 5 Recovery of the $v_{3}\left(\mathrm{SiO}_{4}\right)$ FWHM in zircon as a result of electron irradiation. A: Charts showing the decrease of the FWHMs at irradiated spots as well as reference values (from nonirradiated sample areas). Outlier data points are shown as transparent columns. b and c Decrease of the FWHMs at irradiated spots as well as reference values (placed around the back edges) shown on surface plots with proportional current and time axes. Outlier data points are replaced by the arithmetic mean of the FWHMs in adjacent data points. In $\mathbf{b}$, the set of plots was scaled in $z$ to minimum and maximum FWHM values in each sample, while in $\mathbf{c}$ a common $z$ scale was employed encompassing the FWHM range of all samples (except syn). 

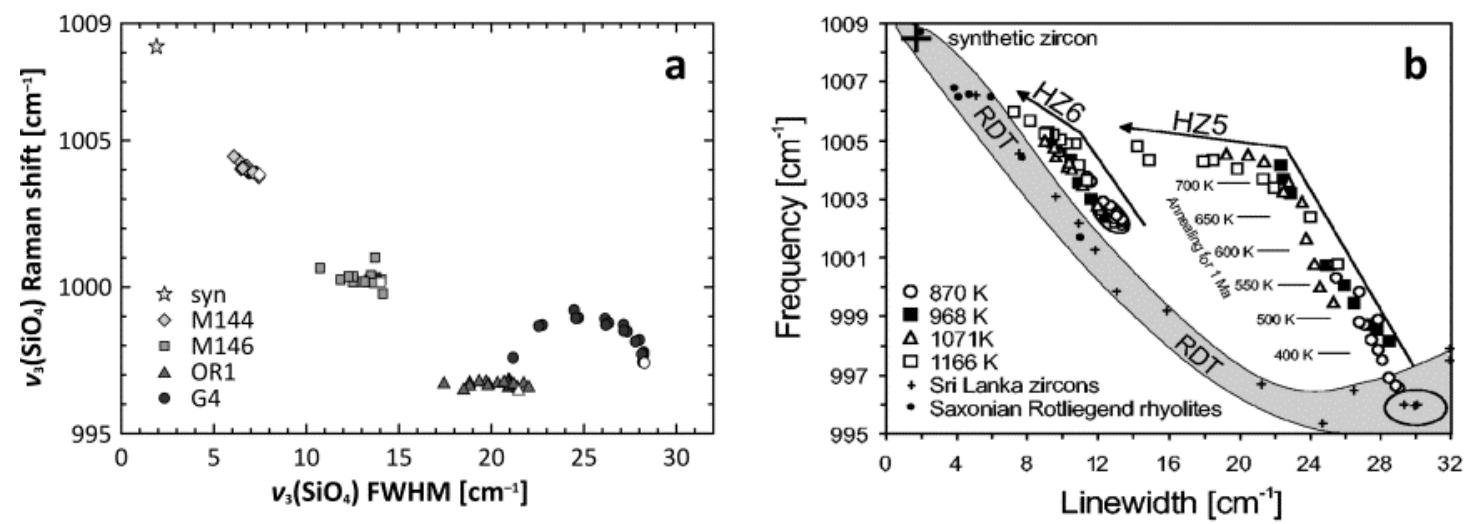

Fig. 6 a Changes of Raman parameters caused by electron-beam annealing. Open symbols: data obtained from non-irradiated samples. b The evolution of position vs. band width during thermal annealing (reproduced from Geisler et al., 2001, with permission by E. Schweizerbart Science Publishers, http://www.schweizerbart.de)
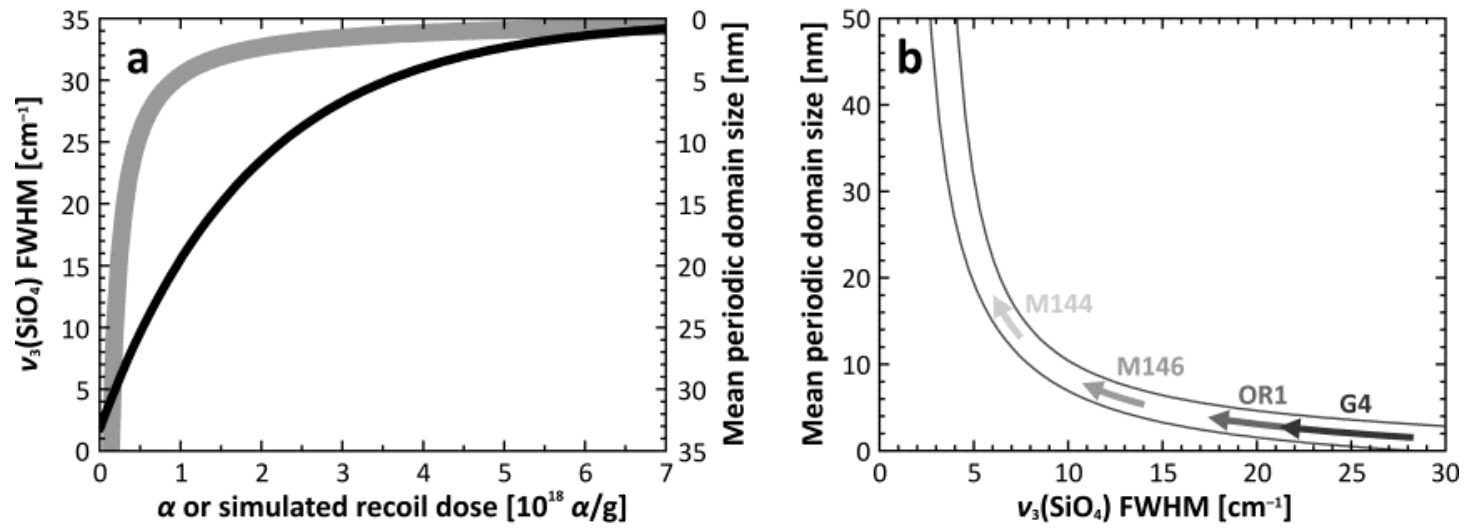

Fig. 7 The relationship between zircon $v_{3}\left(\mathrm{SiO}_{4}\right)$ Raman FWHM (data of Palenik et al. 2003, recalculated using Eq. 2) and the mean periodic domain size, inferred from a recoil damage connectivity model (Eq. 3; after Ketcham et al., 2013). a Raman FWHM (black) and estimated mean periodic domain size (grey) as a function of calculated $\alpha$ dose. b Estimated mean periodic domain size as a function of the Raman FWHM. The properties of the samples in this study before and after electron irradiation are marked with arrows. The thickness of the line suggests large inferred uncertainties in this relationship 